\title{
Smart Sensors and Virtual Physiology Human Approach as a Basis of Personalized Therapies in Diabetes Mellitus
}

\author{
Carlos M. Fernández Peruchena ${ }^{\#}$ and Manuel Prado-Velasco*,\#
}

\author{
Multilevel Modelling and Emerging Technologies in Bioengineering (M2TB) Research Group, University of Seville, \\ Spain
}

\begin{abstract}
Diabetes mellitus (DM) has a growing incidence and prevalence in modern societies, pushed by the aging and change of life styles. Despite the huge resources dedicated to improve their quality of life, mortality and morbidity rates, these are still very poor. In this work, DM pathology is revised from clinical and metabolic points of view, as well as mathematical models related to DM, with the aim of justifying an evolution of DM therapies towards the correction of the physiological metabolic loops involved. We analyze the reliability of mathematical models, under the perspective of virtual physiological human (VPH) initiatives, for generating and integrating customized knowledge about patients, which is needed for that evolution. Wearable smart sensors play a key role in this frame, as they provide patient's information to the models.

A telehealthcare computational architecture based on distributed smart sensors (first processing layer) and personalized physiological mathematical models integrated in Human Physiological Images (HPI) computational components (second processing layer), is presented. This technology was designed for a renal disease telehealthcare in earlier works and promotes crossroads between smart sensors and the VPH initiative. We suggest that it is able to support a truly personalized, preventive, and predictive healthcare model for the delivery of evolved DM therapies.
\end{abstract}

Keywords: Diabetes mellitus, personalized therapies, metabolic syndrome, multilevel physiological modeling, virtual physiological human, digital healthcare, EHR.

\section{INTRODUCTION}

Glycemia in humans must range from 70 to $110 \mathrm{mg} / \mathrm{dl}$. Although exogenous factors may alter this concentration (food intake, digestion, or exercise), there are pancreatic endocrine hormones (mainly insulin and glucagon), that maintain this level [1]. The increase of glycemia is the main effect associated with diabetes mellitus (DM).

Two general types of DM have been classically considered, type I and II. These were called commonly as Insulin-dependent DM (IDDM) and non-insulin-dependent DM (NIDDM), for types I and II respectively [2]. This classification could be considered proper in earlier stages of type II DM, characterized by high plasmatic insulin concentration due to the insulin resistance and the capability of $\beta$ cells to secrete insulin. However, despite physical exercise, as well as pharmacological treatments to promote the increase of insulin sensitivity (e.g. thiazolidinediones or biguanides), in later stages of type II DM, $\beta$ cells are unable to produce enough insulin and the previous division IDDM NIDDM turns misleading. This is particularly important since type II DM occurs in $90-95 \%$ of cases in the world [3]. In spite of this lack, this terminology was proposed by the WHO in 1980 and kept in a subsequent meeting in 1985,

\footnotetext{
*Address correspondence to this author at the Multilevel Modelling and Emerging Technologies in Bioengineering (M2TB), Research Group, Departamento de Ingeniería Gráfica, Escuela Superior de Ingenieros, Universidad de Sevilla, C. de los Descubrimientos, s/n , 41092 - Sevilla, Spain; Tel: 034 954486160; Fax: 034 954486158; E-mail: mpradov@us.es

"Authors contributed equally to this work.
}

with several additions like gestational DM (GDM), impaired glucose tolerance (IGT) and others (for the remaining) [4]. Therefore, classification of 1985 was a compromise between clinical and etiologic perspectives.

The elusive character of DM was explicitly pointed in subsequent recommendations, by the American Diabetes Association (ADA) in 1997 and WHO in 1996 and 1999 [4]. According to this last classification, type I DM is caused mainly by destructions or impairment of pancreatic $\beta$ cells, whereas type II DM is associated with deficiencies in insulin secretion (including hyperinsulinemia) due mainly to a decrease on insulin sensitivity. This new classification tries to include also several clinical stages of DM, and therefore it is kept the IGT and it is added the impaired fasting glucose (IFG). The IGT and IFG are considered previous to DM, and thus they are taken as risk factors. Gestational DB is also kept and completed with gestational IGT.

The foundation of this current classification is indeed more complex, because it tries to include also etiologic factors. Moreover, many clinical studies claim that there is a need to review preestablished concepts regarding DM. Causal analyses and relationships among physical exercise, dyslipidemia, insulin resistance, and metabolic syndrome are key concepts in this task $[5,6]$.

A decade after last recommendation from ADA and WHO, current scientific knowledge, together with poor advances in the quality of life of DM patients, suggest that the causal relationship: insulin deficiency (cause) metabolic syndrome (effect) is faulty. 
Traditional DM therapies are focused to achieve a normal glycemia (as the increase of blood glucose concentration is the main effect associated with DM) by means of diet control, physical exercise and life style, and drugs. The most used drug is insulin, excepting in the first stages of type II DM. Nonetheless, DM patients die mainly due to cardiovascular events, renal insufficiency complications, and other complications of DM that are not directly induced by glycemia.

World population suffering DM was 167 millions in 2000 and projections for 2030 surpasses 350 millions [7, 8]. Mortality rates in DM populations are similar to those ones of HIV patients [7]. Growing prevalence and incidence justify why this pathology is one of the main targets in the ehealth area. Many studies have demonstrated that the improvement in knowledge discovering and accessing is key in the management of chronic pathologies [9]. Therefore, digital healthcare will evolve towards knowledge-based paradigms based on a richer Electronic Health Record (EHR).

A relevant case of a clinical information management system with ability for taking clinical decisions, focused to $\mathrm{DM}$ and end stage renal disease (ESRD), was presented in [10]. One of the strong points of that study was the integrative character of the e-health system, coupling the clinical decision system with the information workflow of the healthcare model. Many subsequent studies have confirmed the necessity to join those features for the success and adoption of the e-health systems. The authors presented also a methodology based on system modeling for the design and evaluation of this type of systems. A main goal of that ehealth system was the control of glycemia. This one has been kept as the main objective of near all the following e-health systems for DM patients.

A wealth of research and innovative efforts has been developed to improve the quality of life of DM patients by means of digital telehealthcare. Results obtained in a Randomized Controlled Trial (RCT) study during 9 months carried out with type I DM patients aged 18 - 30 years, did not show any significant difference between intervention and control groups [11]. The digital healthcare system was based on a mobile platform (phone + blood glucose monitor) with option for recording insulin dose, food intake, and activity levels. Differences between intervention and control were due to the stronger feedback and counsel received by the intervention group. Other RCT study carried out during one year over 1665 type II DM patients, aged 55 years or greater and included in the Medicare system (USA), demonstrated an improvement of glycemia and lipid control, although it was very slight (e.g. HbA1C reduction was $0.18 \%$ greater in the intervention than in control group). This was achieved through a modern digital homecare system able to monitor plasma glucose and blood pressure remotely, and to provide videoconference, clinical data access, and educational resources to patients, specialized nurses, and other professionals. Other systems are oriented to the diagnostic, prevention, and care of complications associated with DM [12].

In summary, huge research and innovative efforts have been performed to improve the quality of life of DM patients by means of digital telehealthcare. Although clinical studies show that patients reduce complications if glycemia control is improved, it is now clear that therapies focused only on that goal are unable to avoid them. Despite this fact, digital healthcare systems continue their orientation almost uniquely towards the control of glycemia. The current scenario suggests that it is necessary a new road for treatment and prevention in DM and other chronic pathologies.

In this work, it will be depicted a multilevel control perspective of human metabolism, with the aim of showing several of its main features involved in the DM pathology. This analysis will provide clues that will drive the evolution of the DM therapies towards the correction of the physiological metabolic loops involved. Some technological aspects of a computational architecture of e-health designed for assisting ESRD patients, which is able to support this evolution, will be briefly presented and discussed.

\section{METHODOLOGY}

The article presents a new personalized therapy approach for DM care. The key aspect of this one is the knowledge of the specific etiology of each DM patient. The precise knowledge of each DM etiology is linked to a metabolic disorder. As a consequence, the article depicts a concise description of human metabolism related to DM under a multilevel perspective, taking into account the importance of control mechanisms and their personalized and adaptive character. It is revised also the role of insulin to justify its involvement on the current clinical standpoint of DM.

The article shows subsequently some mathematical models of different aspects and organizational levels of the human metabolism related to DM. Those mathematical models can be linked to the Physiome initiative. The latter is a comprehensive framework for modeling the human body using computational methods that can incorporate the biochemistry, biophysics and anatomy of cells, tissues and organs. The Virtual Physiological Human (VPH) is a similar initiative that tries enabling collaborative investigation concerning the human body as a complex system, within the Seventh Framework Program of the European Commission. DM mathematical models are reviewed under the perspective of these initiatives.

This is followed by a review of the healthcare and quality of life (QoL) of DM patients.

Previously to the presentation of a new approach for ehealth based on the fusion of VPH with smart sensors, it is shown a case study illustrating the main differences between an etiology-based treatment, and a treatment based only on the correction of hyperglycemia. The case study exposed shows a particular DM where the proper knowledge of the etiology has allowed the design of a personalized treatment that corrects the physiological loops.

The new healthcare approach presented in this article makes use of concepts developed and evaluated in earlier works.

\section{A MULTILEVEL CONTROL PERSPECTIVE OF METABOLISM FOCUSED TO DIABETES}

The following lines present some relevant aspects and mechanisms of human metabolism taking insulin as a pivot, with the aim of analyzing its role in the current clinical 
perspective of DM. The review tries to throw light on the evolution of new treatments and healthcare processes associated with DM, under a bioengineering perspective.

Feedback and feedforward are central in metabolic processes. Metabolic control is driven mainly by enzymes, although only a percentage of chemical reactions involved in metabolism are really regulated, in order to maximize the efficacy of the whole system. The most common control mechanism is allosteric due to the natural relationship between this type of behavior and feedback and feedforward loops [13]. Both types of loops have been deeply studied in systems engineering and there is a wide and powerful mathematical and methodological framework to address with them.

An additional property in metabolic control is the compartmentalization of processes. This property of living systems allows using enzymatic proteins for different objectives as a function of their placement. Compartmentalization requires the presence of membranes or separation surfaces, able to decide which substances pass through, as well as marking protocols to signal the activation (e.g. conformational change by peptide addition). Signaling and communication are not limited to cellular dynamics, but they act on different cell groups (cell structures) and levels, in agreement with functional organizations, such as nephrons in kidneys or islets of Langerhans in pancreas.

Human physiology involves the integrated regulation of all these systems at all levels. Important concepts such as robustness, adaptability, modularity and functional emergence in living systems can be mathematically analyzed under this multilevel regulatory perspective [14-16].

It is usual to divide human metabolism into three great paths related to carbohydrates, lipids, and proteins. These paths are functionally connected at many places and levels in the whole regulatory human physiological system, as we try to show in this Section, and will justify a key conclusion: Under the unknown etiology of most DM scenarios, the preconceived notion of a defect in insulin - glycemia subsystem as the cause of DM, should be surpassed.

From a systemic point of view, the pancreas plays a key role in physiology regulation by secreting insulin and glucagon. There are other important hormones secreted by pancreas such as amylin or somatostatin, although their functions are less known and they exceed the objective of the present analysis.

Carbohydrates are transformed in oligosaccharides and hydrolyzed mainly to glucose, which is absorbed through the intestinal tract, and mostly distributed to the liver through portal vein. Liver is the main controller of glucose systemic circulation. Glucose is converted and stored in glycogen mostly in liver. If the storage capacity of liver is exceeded, then glucose is converted to triglycerides that go away as very low lipoproteins (VLDL). This issue points to one link between carbohydrates and lipids paths. Other important connections among the three great metabolic paths aforementioned will be subsequently emphasized.

Glucose is absorbed by tissues thanks to different glucose transporters. Liver and gut cells have GLUT-2 transporter (high capacity transporter), with a high Michaelis-Menten constant $K_{m}$, which avoid the saturation of these ones despite high glucose concentrations. GLUT-2 is also in pancreatic $\beta$ cells to assure a proper sensitivity (and even linearity) of this organ to a wide range of glucose concentrations. Muscle and fat tissue have GLUT-4 glucose transporter, which impedes the use of glucose with low plasma concentrations. If this situation occurs, myocytes use fatty acids, and fat cells inhibit the storage of lipids. Other tissues have cells with GLUT-1 or GLUT-3 transporters, characterized by a low $K m$, which allows the use of glucose even with low concentration. Neurons and glia are key examples.

The effect of insulin on the cell metabolism is mediated by the insulin receptor into cell membrane. Fig. (1) shows a simplified block diagram of this one.

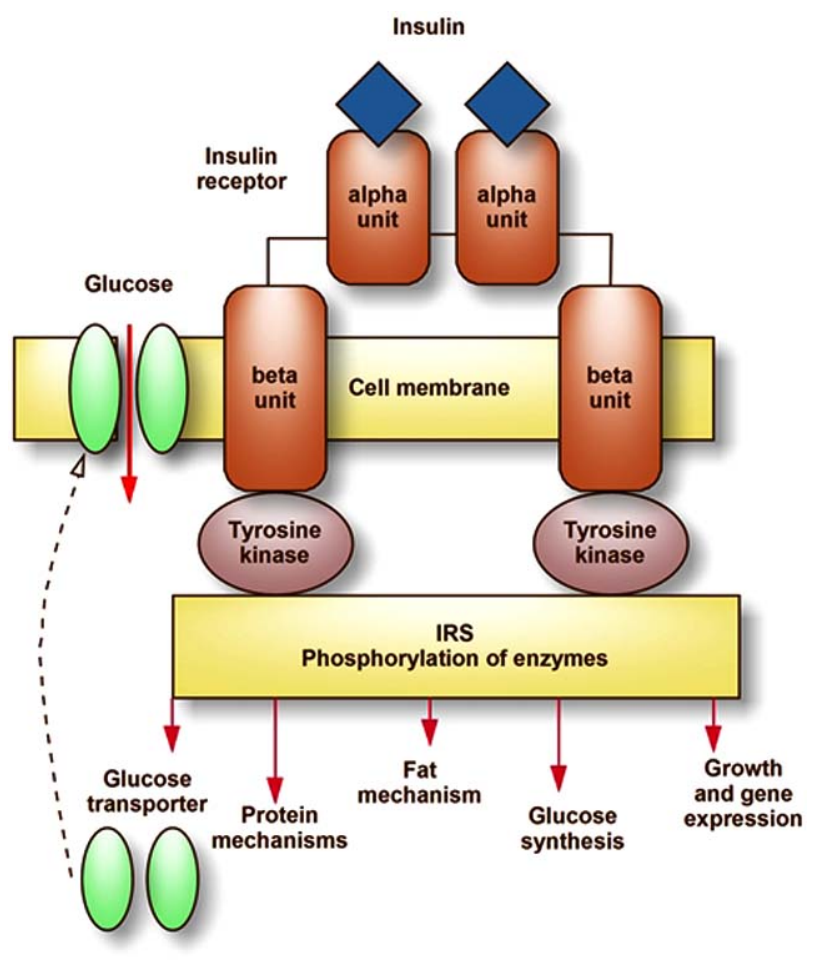

Fig. (1). Block diagram of insulin receptor together with associated IRS and indications of regulated molecular machinery. Adapted from [2].

The activation of this molecule, composed by four subunits, two $\alpha$ and two $\beta$ units, promotes the autophosphorylation of $\beta$ subunits, which activate local tyrosine kinases inside the cell, which in turns induce the phosphorylation of intracellular enzymes. An important group of these are denoted insulin-receptor substrates (IRS) [2]. Their expression depends on the tissue. It is remarkable to underline that insulin acts simultaneously on the three major metabolic paths through this insulin receptor. Therefore, insulin receptor set up another important link among proteins, lipids, and glucose mechanisms, which in addition depends on the cell type, and thus on the functional organization.

From a control point of view, insulin appears as a systemic messenger that acts in feedforward loops (from upstream substrates) into different organization levels. Its action is modulated by the type of cell via expressed IRS. It can also act in a direct way into several subcellular levels, 
modulating feedback loops (from downstream products). Some details concerning this issue are clarified in the following subsections.

\section{Carbohydrate Mechanisms}

Insulin promotes the absorption of glucose into myocytes mainly in two situations: after meals and during moderate and strong physical exercise. During exercise, muscle fibers turn more permeable to glucose, even with low concentration of plasmatic insulin. In other situations myocytes use fatty acids. This behavior involves different mechanisms at different levels.

Glucose 6-phosphate (6-P) is the starting point of near all metabolic paths related to carbohydrates. These are known as glucose-6-P pathways, and include synthesis and degradation of glycogen, the pentose phosphate pathway that is needed for the synthesis of the ribose 5-phosphate (base of nucleic acids), and of course the glycolysis pathway, which is the most important (and ancient from a phylogenetic perspective) path of glucose-6-P.

One of the main objectives of glycolysis is to transport the energy from substrates to ATP. The main intracellular regulator enzymes in glycolysis are hexokinase, phosphofructokinase-1 (PFK-1), and piruvate kinase. These enzymes act locally within feedback and feedforward loops, but they are also modulated by insulin and glucagon in a feedforward way (systemic regulation). Insulin promotes the dephosphorylation of PFK-1 to phosphofructokinase-2 (PFK-2). In addition it promotes the dephosphorylation of piruvate kinase, which in such a way can be activated by fructose 1 , 6-bisphosphatase (FBP) to be transformed in piruvate. Piruvate is the main input to the final stage of glycolysis, the tricarboxylic acid cycle or Krebs cycle. This is a key set of chemical reactions in cells of aerobic organisms. In eukaryotes like humans this cycle occurs in the matrix of mitochondrion, in coordination with the oxidative phosphorylation. This way, an increase of insulin or a decrease of glucagon intensifies the activity of Krebs cycle.

A complementary systemic regulation of Krebs cycle occurs via the increase of calcium in the citosol, which in turn promotes its input in the mitochondrion, and the activation of Krebs cycle. In muscle cells, this calcium input is promoted by electrical stimuli implied in muscle contraction. In other tissues like the brain this calcium increase is produced through a second messenger of insulin, the inositol phosphate.

Insulin also increases the activity of glucokinase, one of the main enzymes related to the phosphorylation of glucose to glucose-6-P.

In the liver, insulin induces the inactivation of liver glycogen phosphorylase, which in turn inhibits the transformation of glycogen to glucose. This regulation mechanism connects with gluconeogenesis, a metabolic pathway whose result is the generation of glucose from noncarbohydrate substrates. It is a set of processes that keep glucose concentrations above a minimum value, needed for cells like erythrocytes, retina cells, renal medulla, sexual organs cells, and neurons, among others.

The most important substrates in the gluconeogenesis are lactate, glycerol (which comes from hydrolysis of triglycerides in fat tissue), and even amino acids that can come from muscle proteolysis. Insulin acts also in the systemic regulation of gluconeogenesis via the inhibition of piruvate carboxylase (PC), through transcription mechanisms. The inhibition of $\mathrm{PC}$ reduces the conversion of piruvate to oxaloacetate, the first stage for glucose synthesis. Other hormones, such as glucagon, act in the opposite direction.

This succinct description of some of the carbohydrates pathways shows the multilevel and systemic character of the involved regulation cycles, and underlines how insulin acts by feedforward loops on the main pathways at different organic levels, including the transcriptional system (genetics) and proteins, in different functional cell groups and tissues, and among functional subsystems. Several key connection points among proteins, lipids and carbohydrates pathways have also been shown.

\section{Lipids}

Insulin promotes the use of glucose and reduces the use of lipid in near all tissues. It promotes also the synthesis of fatty acids, especially in the liver, from which they are carried to fat cells as VLDL. This general behavior is supported by a set of metabolic pathways.

Lipid metabolism is conditioned by the solubility of these compounds. Fatty acids, which are lipophilic, circulate in plasma joined to albumin, whereas cholesterol, complex lipids, and triglycerides (amphipathic or lipophilic) do by means of lipoproteins structures, like the aforementioned VLDL. The proteinic component of these compounds is a key element to signaling and marking processes. Moreover, the weak forces that control the binding of different molecules in these aggregates facilitate the interchange of elements with cellular membranes. This last issue is also implicated in the pathophysiology of DM complications like atherosclerosis.

A relevant process of lipid metabolism is the synthesis of triglycerides. This occurs mainly on the liver, intestinal mucosa, and fat tissue. Due to the lack of glycerol kinase on fat tissue, the first precursors needed to the synthesis of triglycerides in this tissue provide from the glycolysis. Therefore, this is another metabolic point where lipids and carbohydrates pathways are coupled.

The inverse mechanism, the hydrolysis of triglycerides to give fatty acids, whose main goal is the oxidative degradation to produce energy, is performed via lipase. This enzyme is activated by glucagon and growth hormone, among others, and inhibited by insulin.

Fatty acids are converted in acetyl-CoA (the number of molecules depends on the fatty acid) by means of several pathways of activation (citosol) and $\beta$-activation (mitochondrion) in many tissues, with the exception of nervous system among others. Acetyl-CoA is an input to the Krebs cycle. The excess of fatty acids pushes an overproduction of acetyl-coA, which due to the sharing use of oxaloacetate, both in Krebs cycle and in gluconeogenesis, promotes the exhaustion of Krebs cycle. A consequence is the conversion of acetyl-coA in ketone bodies.

A low level of insulin promotes the circulation of fatty acids and the gluconeogenesis in the liver. However, in 
pathological conditions, where insulin is low and glucose is high, glycolysis and gluconeogenesis are very active (liver cells have the high capacity glucose transporter GLUT-2). This way, both processes compete by oxaloacetate, resulting in the increase of ketone bodies, which is a typical symptom in patients with type I DM. The associated acute complication is ketoacidosis.

Despite the importance of cholesterol in the pathogenesis of DM, the physiological mechanisms are very complex and not well known. It is known that there are feedback control mechanisms that inhibit the synthesis of low density lipoproteins (LDL) receptors in tissues that use cholesterol, which is synthesized mainly in liver and intestinal mucosa.

With the exception of pathological conditions, the cholesterol ingestion has not a great influence on its plasmatic levels.

\section{Proteins}

Protein pathways are worse known than carbohydrate ones. However, we emphasize here some details that illustrate their coupling and multilevel organization.

Protein metabolic processes in the liver include gluconeogenesis (energy use), synthesis of other nitrogenous compounds, and synthesis of the most important plasmatic proteins. Insulin induces the protein synthesis and storage, as well as the input of amino acids to cells. Growth hormone acts in a synergistic manner with insulin, promoting the amino acid input to cells. This synergy has been experimentally shown in depancreatized and hypophysectomized rats [2], and throws light about the importance to prevent DM during growing stages of humans.

Insulin acts also in other steps of the nitrogenous metabolism. It is a needed factor in the traduction of messenger RNA (mRNA), in such a way that without insulin the ribosomic molecular machinery does not function properly. It also speeds the transcription rate of some DNA sequences, promoting the synthesis of a set of enzymes that affect the storage of carbohydrates, lipids and proteins.

Insulin inhibits also the protein catabolism, in agreement with its anabolic character.

Accordingly, a pathological low plasma insulin concentration induces protein catabolism and liberation of amino acids to blood, increasing the urine urea concentration and many other dysfunctions. This abnormal protein catabolism presents an evocative parallelism with the abnormal protein catabolism in End Stage Renal Disease (ESRD) patients submitted to periodic hemodialysis [17].

\section{Multilevel Dynamics}

Above Sections show clearly the multilevel, nonhomogeneous, and complex character of metabolic regulation in humans. From a systemic point of view, these mechanisms act in a coordinated way to adapt the use of metabolic substrates in each place (cells) to the intake and fasting cycles, physical activity, and environment. The insulin is one of the most important systemic messengers involved, although it is not the unique. It acts mainly by feedforward cycles, although insulin secretion by $\beta$ cells in pancreas requires also a feedback mechanism.
The belief concerning DM as a disease related only to carbohydrates and insulin led the idea that insulin secretion is controlled exclusively by glucose. However different studies have shown that $\beta$ cell insulin secretion depends also on plasmatic amino acids, like arginine and lysine, which acts amplifying the response of $\beta$ cells to glucose. As it could be expected, some gastrointestinal hormones, like gastric inhibitory polypeptide (GIP), act in similar way, promoting an anticipatory mechanism to the increase of glucose and amino acids after meal.

Pancreas $\beta$-cell receptors are sensible also to other hormones involved in metabolic processes, like glucagon, growth hormone, progesterone, and estrogen. There are other factors implied in the secretion response, as $\beta$ adrenergic stimulation and acetylcholine (positive) or $\alpha$ adrenergic stimulation and somatostatin (negative).

Regarding dynamical aspects of insulin, several studies have measured the relationship between plasma concentration and time, after different inputs (e.g. a sudden increase on glucose concentration) [1]. Others show that insulin release oscillates with a period of 3-6 minutes, even in basal conditions, and propose several underlying mechanisms [18]. A more detailed explanation is provided in the following Section. The high oscillations of glucose concentration in the portal vein, from pancreas to liver, together with other observations suggest that these oscillations are clinically relevant. Moreover, disturbances in this dynamics could contribute to insulin resistance in type II DM.

This review suggests that current insulin therapies (bolus or continuous infusion-based) are not able to correct the control loops involved in a personalized manner in each DM. Moreover, current insulin therapies could promote changes and adaptations at different levels of organic functional organizations that degenerate in severe metabolic damages in a long term. Cells and organic structures with lesser abilities to adapt their metabolite pathways to the rigorous physiological conditions associated with this pathology and current therapies, as nervous system or retina cells, will be more exposed to complications.

Damages on multilevel regulatory pathways affect to carbohydrate, proteins, and lipids pathways, independently of the initial etiology of a particular DM. This agrees with the metabolic syndrome underlying DM.

\section{MATHEMATICAL MODELING IN DIABETES MELLITUS}

The prevalence of DM has stimulated research on several aspects related with DM and its complications. Many mathematical models have been developed to describe different aspects of DM at several levels, from $\beta$ cells to insulin-glucose dynamics. The most relevant mathematical models concerning to the physiology underlying DM are briefly shown in this Section. We are interested in the ability of these ones to integrate and extract biomedical knowledge that could be applied in telehealthcare systems.

\section{$\beta$ Cells and Pancreatic Islets}

A huge number of models have been developed to describe $\beta$ cells insulin secretion. These models, in particular those proposed by Licko \& Grodsky [19, 20] and Cerasi 
[21], established a reference framework to describe $\beta$ cells function. Few years later, the first widely accepted mathematical model concerning the individual function of $\beta$ cells was published [22].

The Model proposed by Topp [23] is composed of three non linear ordinary differential equations as follows:

$$
\begin{aligned}
\frac{d G}{d t} & =a-(b+c \cdot I) \cdot G \\
\frac{d I}{d t} & =\frac{d \cdot \beta \cdot G^{2}}{\left(e+G^{2}\right)}-f \cdot I \\
\frac{d \beta}{d t} & =\left(-g+h \cdot G-i \cdot G^{2}\right) \cdot \beta
\end{aligned}
$$

Where plasma glucose concentration (G) is in $\mathrm{mg} / \mathrm{dl}$, plasma insulin concentration (I) is in $\mu \mathrm{U} / \mathrm{ml}$, and $\beta$ cell mass $(\beta)$ is in $\mathrm{mg}$.

The biological interpretation of parameters involved is shown in Table $\mathbf{1 .}$

Table 1. Biological Interpretation of Parameters Involved in Topp Model

\begin{tabular}{|c|c|}
\hline Parameter & Biological interpretation \\
\hline \hline a & liver glucose production rate $(\mathrm{G}=0)$ \\
\hline $\mathrm{b}$ & Insulin-independent rate of glucose elimination \\
\hline $\mathrm{c}$ & Insulin-induced glucose absorption rate \\
\hline $\mathrm{d}$ & Maximum $\beta$ cells insulin secretion rate \\
\hline $\mathrm{e}$ & Sigmoid turning point \\
\hline $\mathrm{f}$ & Whole-body insulin elimination rate \\
\hline $\mathrm{g}$ & $\beta$ cells natural death-rate \\
\hline $\mathrm{h}, \mathrm{i}$ & Allow the determination of $\beta$ cells tolerance range \\
\hline
\end{tabular}

Topp's model allows a transition from physiological equilibrium state to a hyperglycaemic one. Furthermore, it predicts that some defects in glucose and/or insulin dynamics can increase glucose at a higher level than $\beta$ cell mass adaptation rate. It provides a $\beta$ cell mass of $300 \mathrm{mg}$, which is not a valid physiological value, since human $\beta$ cell mass is normally about $850 \mathrm{mg}$.

Hernandez introduced a modification for this model [24], through the consideration of insulin receptor dynamics:

$$
\begin{aligned}
\frac{d G}{d t} & =a-(b+c \cdot R \cdot I) \cdot G \\
\frac{d I}{d t} & =\frac{d \beta \cdot G^{2}}{(1+R) \cdot\left(e+G^{2}\right)}-f \cdot I-f \cdot R \cdot I \\
\frac{d \beta}{d t} & =\left(-g+h \cdot G-i \cdot G^{2}\right) \cdot \beta \\
\frac{d R}{d t} & =j \cdot(1-R)-k \cdot I \cdot R-l \cdot R
\end{aligned}
$$

$\mathrm{R}$ is the fraction of insulin receptors in cell muscle surface. Insulin receptor dynamics is represented through the following parameters: $\mathrm{j}$ (insulin-receptor recycling rate), $\mathrm{k}$ (insulin-dependent receptor endocytosis rate), and 1 (insulinindependent receptor endocytosis rate).
Hernández model improved quantitative results, providing a physiological value of $\beta$ cell mass, associated with experimental receptor dynamics. It predicted, under normal conditions, basal levels of $\beta$ cell mass, glucose and insulin dynamics, and insulin receptors, similar to those found experimentally. The model shows how $\beta$ cell mass control malfunction (represented by the parameters $\mathrm{g}$, $\mathrm{h}$ and i) can lead to diabetes mellitus, either creating hyperglycaemic glucose levels or causing a saddle-node bifurcation that leaves the pathological state as a global attractor.

Different studies have shown that physical exercise can increase insulin sensitivity in a 36\%, which allows a decrease in insulin levels required to maintain glucose concentration. Reduction in insulin equilibrium levels implies a lower $\beta$ cell mass requirement and an increase in cellular insulin receptors. However, a sedentary way of life (accompanied by obesity) can reduce insulin sensitivity by $50-100 \%$. Hernandez model predicts an insulin-resistant state for a reduction of $60 \%$.

However, these models do not consider other hormone secreting cells (such $\alpha$ and $\delta$ cells in the islets of Langerhans), which contributes to regulate glucose and insulin levels. Furthermore, the consideration of an upper bound on $\beta$ cell mass is desirable in these models, as well as the incorporation of insulin sensitivity dynamics.

It is worth to remark in this Section the consensus model for glucose-stimulated insulin secretion [18]. In this model, ATP (generated by mitochondrial metabolism) promotes closure of $\mathrm{K}_{\text {ATP }}$ channels. The depolarization of the plasma membrane induces the opening of $\mathrm{Ca}^{2+}$ channels increasing the cytosolic $\mathrm{Ca}^{2+}$ concentration, triggering insulin exocytosis. Nonetheless, the $\mathrm{Ca}^{2+}$ signal alone cannot explain either sustained secretion [25] or the pulsatile nature of secretion. As a consequence, the consensus model does not explain glucose-stimulated insulin secretion dynamics. In subsequent studies, the consensus model mechanism has been included in a more general dynamics frame [18].

Current models show a convergence between different hypotheses (electrical activity and glycolysis) that explain oscillations in the secretion of pancreatic islets. Both mechanisms can work in a cooperative manner to produce the rhythm secretion of insulin in a period of 5 minutes observed experimentally in vivo. The key role in the formation of oscillations is attributed to the allosteric enzyme phosphofructokinase-M (PFK-M) (PFK isoform in muscles), which is activated by physiological micromolar concentrations of FBP and adenosine monophosphate (AMP), and inhibited by ATP.

Bertram and Sherman proposed recently a pancreatic islets model, where electrical activity (bursts) was attributed to a cytosolic $\mathrm{Ca}^{2+}$ feedback on ionic channels. This mathematical model reproduces the slower oscillations of insulin release by a metabolic subsystem driven by glycolysis [26]. The interaction of glycolytic and $\mathrm{Ca}^{2+}$ insulin release dynamics can result in a wide variety of rhythmic behaviours in pancreatic islets, in agreement with experimental results.

\section{Systemic Modeling}

Mathematical modeling of diabetes has focused mainly to the dynamics of glucose and insulin levels in a temporal 
scale of minutes [27]. Generally, these models are used as tools to measure rates (production and absorption of glucose and secretion of insulin), or sensitivities (insulin sensitivity or glucose effectiveness). Most of these models are dedicated to glucose-insulin dynamics, pointing to a lack of interest in the consideration of other relevant aspects of the metabolism.

In 1939, Himsworth and Ker introduced the first approach to measure insulin sensitivity in vivo [28]. Later, several mathematical models were proposed in this area, being a remarkable example the one proposed by Bolie in 1961 about blood glucose regulation [29]. Nevertheless, the factual beginning of glucose-insulin dynamics modelling is determined by the publication of the minimal model by Bergman and Cobelli [27]. Since the publication of the minimal model, their authors have published a number of works completing, revising or validating it. Several authors have introduced modifications to the minimal model, being remarkable the one proposed by Derouich and Boutayeb, which introduced parameters related to the physical exercise [30].

However, the minimal model and subsequent derivations show several drawbacks. For example, it considers the glucose-insulin system alone, neglecting that it is only part of a complex metabolic regulation network, as described in the previous Section. Furthermore, some of the mathematical results provided by the model are not realistic, and it is necessary the introduction of an artificial variable to account for the delay in the insulin action.

To overcome some of these limitations, De Gaetano and Arino proposed a differential delay in the insulin action in their dynamical model $[31,32]$. Other authors have shown the ability of this model to allow the simultaneous determination of insulin secretion and glucose absorption [33]. The dynamics of glucose-insulin has been described also by means of optimal control strategies [34].

The mathematical description of insulin distribution in physiological compartments presents also many difficulties. The main trouble in the case of subcutaneous insulin kinetics resides in the distribution of subcutaneous reservoirs and their transport to plasma. Several models have been proposed for addressing this issue: Kobayashy (M1), Kraegen (M2), Puckett (M3), Shimoda (M4), Berger (M5) and Trajanoski (M6) [35]. Their approaches include lumped parameter modelling methodologies (M1, M2, M3, M4), empirical description of the subcutaneous insulin absorption based on published data (M5), and physicochemical distributed-parameter models (M6). A review of the main modelling and simulation methodologies that facilitates the interpretation of this taxonomy appears in [36].

\section{Integration and Extraction of Knowledge from DM Mathematical Models}

In the last decades, a number of mathematical models have been published describing different aspects of DM. We have shown several approaches at different levels, which could be integrated under a proper multilevel modeling methodology. Marée highlights the importance of modeling individual cells to explain the beginning of diabetes [37], as they are constituent parts of higher functional level models.
Due to the inherent complexity of biological systems, the development of mathematical models to describe them is a complicated task. One of the most successful approaches to develop them is based on their hierarchical organization. Accordingly, a model can be constructed by means of the connection of simpler models which describes phenomena at low levels [38]. Models can represent different parts of a system at different levels, allowing their integration in order to provide the behaviour of the whole system. This fact is a key point in the approach of DM modelling, due to the wealth of multilevel control loops involved.

The integrative approach is nowadays a key aspect in mathematical modelling of biological systems. Multilevel modelling in the frame defined by the Physiome project is possible through the connection of models with appropriate tools. Among these tools, we can highlight here ERATO System Biology Workbench (SBW), a framework that allows both legacy and sharing resources at different levels [39]. However, this type of integration is not possible in the case of strongly coupled models, because dynamics is distorted.

Multilevel modeling approach applied to diabetes is a promising tool to study the whole system behavior and also to investigate the effects of specific malfunctions on its global behavior. These models must connect the dynamics of $\beta$ cell mass with the dynamics of insulin and glucose, within the human metabolic network.

In a telehealthcare system, these multilevel models describing different aspects of DM can constitute a key knowledge generation layer [40]. There are some linking points between this layer and the Evidence Based Medicine (EBM) approach. EBM symbolizes the rational, explicit, judicious and current use of the best scientific evidence concerning treatments and outcomes in different pathologies [41]. Unfortunately, there is a lack of methodologies able to address EBM sources through expert systems.

\section{QUALITY OF LIFE IN DIABETES MELLITUS: A SUCCINCT REVIEW}

Several techniques to evaluate the quality of life (QoL) of DM patients have been developed. One of the first specific evaluation methods to quantify the QoL related to DM treatments was proposed in 1988 [42]. This method is commonly known as DQOL (Diabetes Quality Of Life), and assesses five general aspects related with DM and its treatment.

Several researchers have projected other classification methods [43-45]. Nowadays, the most accepted classification method is the one based in the Medical Outcome Survey [46]. This method assigns a higher score to higher QoL, in a scale ranging from 0 to 100 .

It is generally accepted that DM patients suffer an important decrease in their QoL [47]. The quality of life of DM patients is deteriorated due to physical and emotional DM effects, and several fundamental aspects of human behavior are affected, like work activity or family life.

Most important complications of DM are classified in acute and chronic. Acute complications include ketoacidosis (DKA), and non-ketosis hyper-osmolar state (NKHS), but chronic complications are the main responsible of the high 
mortality rate and low QoL of DM patients and they have effect on most of the organs $[3,7]$. We can divide chronic complications into vascular and non-vascular complications. First ones are classified into microvascular (retinopathy, neuropathy, and nephropathy) and macrovascular (coronary artery disease, peripheral vascular disease, cerebrovascular disease). Non-vascular complications include gastroporesis, sexual dysfunction, and skin changes.

Life expectancy of DM patients is 10 years lesser than the average. A $72 \%$ of type II DM patients suffer at least one derived complication and $24 \%$ undergo both microvascular and macrovascular complications. Only $7 \%$ of the healthcare expenditure is due to insulin and other oral drugs, whereas $55 \%$ is due to hospitalization [48]. Near $75 \%$ of subjects with DM for more than 15 years undergo diabetic retinopathy, whereas $50 \%$ of DM patients have some degree of neuropathy [3]. DM patients present also much higher prevalence than the average population of cardiovascular diseases, stroke, and hypertension. Infections are more frequent and severe in this population. Furthermore, nonhealing ulcers that can lead to non-traumatic amputation are frequent when the loss of peripheral sensation (polyneuropathy) is coupled with impaired microvascular and macrovascular junction in the periphery.

Many subjects present DM complications when this disease is diagnosed, in spite of many studies have shown that DM can be detected in earlier stages. This is because prevention methods are hardly deployed by the health system. To improve the detection of DM (without clinical symptoms), the WHO and ADA recommended a combined use of IFG and IGT [3].

Moreover, prevention and detection methods of DM lack of an integrative and personalized view, and therapies are gross and non-personalized.

Nevertheless, in spite of the disappointing situation given by these facts and the growing prevalence and bad QoL, mortality, and morbidity rates of DM patients, advances in some monogenic DM types have been achieved. It is worth to highlight here the Maturity-onset diabetes of the young (MODY) and the neonatal diabetes case, as a case study of successful treatment. MODY and neonatal diabetes are $\beta$-cell disorders, and they are currently recognized as a set of discrete subtypes defined by a genetic etiology.

In particular, MODY is considered a DM different from type I and II, although it is usually diagnosed as one of them. MODY is not insulin dependent, in opposition to Neonatal diabetes, which is insulin dependent and diagnosed in the first 3 months of life. The most common permanent neonatal diabetes (which required continual insulin treatment) is due to a mutation in the KCNJ11 gene encoding the Kir6.2 subunit of the $\beta$-cell $K_{\text {ATP }}$ channel [49]. This channel controls insulin secretion in pancreatic $\beta$-cells. Most of patients with heterozygous mutation Kir6.2 were treated by insulin administration until the etiology was known. Afterwards, it was tested the possibility to induce insulin secretion by means of sulfonylureas, taking profit that they bind to the sulfonylurea receptor 1 of the $\mathrm{K}_{\text {ATP }}$ channel and close the channel through a route independent of ATP.

Different physiological and clinical studies confirmed this hypothesis with successful outcomes. Studies confirmed also the normalization of glucose in the long-term (by HbA1c), as well as the reduction of hypoglycemic events with respect to insulin treatment. Continuous monitoring during 24 hours showed also a good control of glycemia during meals [50-52]. Moreover, a recent work indicates that glycemia is recovered when switching from insulin treatment to sulfonylurea even in adult patients with permanent neonatal diabetes associated with KCNJ11 mutation [53].

Despite we are aware that neonatal DM is a very particular case in this pathology, it has been presented with the goal to illustrate the difference between a treatment based on the etiology and a treatment designed to correct the main homeostatic dysfunction, that is, hyperglycemia. From a physiological point of view, the treatment shown in the case study is able to restore regulatory loops, whereas current insulin treatments do not.

\section{VPH AND SMART SENSORS IN THE FOUNDATION OF A NEW TELEHEALTHCARE PARADIGM}

The treatment of the case study depicted in the previous Section tries, in essence, to reestablish physiological control loops. The advantages of this approach have been clarified in Section 3. The main handicap of this one it is the necessity of a better knowledge of the physiological basis of each patient etiology. A methodology and technology that addresses in a synergistic manner the discovering of causal mechanisms of metabolic syndrome, as well as the use of this knowledge to advance towards personalized treatments that correct physiological control loops, is presented in this Section.

\section{E-Health and Smart Sensor-Based Supervision}

Health personalization has become a main objective in the development of e-health systems. To achieve it, continuous and ubiquitous biosignal monitoring has acquired a key role. Here we propose an integrative and personalized approach for e-health systems that is founded partly in smart sensors. A common attribute of smart sensors is their reactive character, which is accomplished by adapting them to environmental stimuli thorough signal processing algorithms, adaptive control and intelligent decision-making.

The effective integration of the different elements that comprise a sensor system can accomplish behaviour similar to that observed in biological systems, using available energy in the most efficient way. These systems regulate their internal parameters in order to response to changes in environmental conditions. This ability is an essential aspect of biological organisms.

Synergistic approaches among engineers that develop smaller and more integrated adaptive technologies, biologists that discover increasingly selective and sensitive living mechanisms, and material scientists, are producing new ways to build smart sensors [54].

An example of smart sensor in e-health is presented in [55]. It shows a novel system for the analysis of human movement and detection of physical risk activities on the elderly, using smart sensors with ambient intelligence, multimodal capacity, and reactivity to the environment, within a wearable distributed computing architecture. A key element in this architecture is the IASP (intelligent 
accelerometry sticking plaster), which performs accelerometer signal processing and transmits re-sampled signals and impact warnings to a WBS (wearable station base). The WBS is connected to a Smartphone that performs a deeper signal analysis as requested. The two-layer processing architecture of the wearable multimodal smart sensors allows the achievement of hard functional specifications, as is the personalization and real time learning, overcoming limitations of earlier human movement monitors. A previous work demonstrated how the efficiency of a smart movement sensor is clearly improved when it is personalized to the user [62].

\section{E-Health and Model-Based Integrative Research}

VPH is a European initiative that derives from Physiome. The latter started in the middle of 90's with the aim of facilitating collaboration, distributed access, and integration of biomedical knowledge at different organizational living levels [56-58]. Physiome and VPH programs address the description of biological and physiological systems by means of mathematical models whose main common point is that they are not data-driven. These models can use deterministic or stochastic approaches, continuous or discrete dynamics, and lumped or distributed processes. However, their nature is predictive by nature, as opposition to data-driven models [59]. Data-driven models are built by fusing experimental data (e.g. training) with a methodology able to organize the model's output space according to these data. Relevant examples of data-driven models are neuronal networks, regression models, rules-based expert systems and support vector machines.

Physiome and VPH address with advanced concepts of complex systems dynamics, such as multilevel feedback loops, adaptation, non-linearity, emergence of organization levels, and robustness to perturbations [16]. They appear as initiatives whose ultimate goal is to allow scientists and medical practitioners understanding and applying knowledge regarding the causes of diseases from low levels (molecules) to the highest systemic level of an organism.

One of the challenges of the Physiome and VPH initiatives is the development of standards for the exchange of information between models. This task requires formal ontologies to use a standard nomenclature for model components. Several ontologies have been developed in this context (Gene Oncology, Foundation Model of Anatomy, Open Biological Ontologies). They have incorporated in markup language standards for modelling systems to assure the semantic interoperability. Therefore, information at different spatial scales and levels can be consulted using defined ontologies and models hosted in Web databases and encoded in markup languages [60].

Another challenge of the Physiome and VPH initiatives is to develop open source tools for creating, visualizing, and running model simulations [60]. The tools developed in this goal are divided into the following categories: creating and editing ontologies (Protégé), creating and running electrophysiological (CESE, iCell, MozCellMl) and pathway models (CellMLeditor/MozCellML, Gepasi, SBW), creating, running and visualizing anatomical models (BioPSE, cmgui/CMISS, Continuity, SCIRun, Virtual Cell), systems physiology (JSIM), and communication between models (SBW).

A number of virtual human modelling projects are being developed. Among these, we can highlight the following ones: Visible Human (human anatomy, funded by NLM), IUPS Physiome (multi-scale modelling), US Physiome (links to models), Virtual Soldier (wound simulation, funded by DARPA), Virtual Astronaut (Medical education, funded by NASA), EU BioSim (pharmacogenetics, funded by European FW 6), and Living Human (biomechanics, funded by European FW 6) [60].

The link between e-health and integrative research based on networks of mathematical physiological models like those ones promoted by Physiome and VPH is recent. One of the first e-health systems that promotes this connection was presented in [61], and a preliminary proof of concept was published in [62]. The capability of integrative research initiatives based on VPH and Physiome to provide a complementary approach to Predictive, Personalized, Preventive and Participatory (4P) Medicine has been more recently discussed in [63].

Although the literature hardly mentions the connection between e-health and Physiome, there are an increasing number of projects connecting e-health with different artificial and ambient intelligence systems. In this context, we can highlight Tratamiento 2.0 (http://www.indra.es/web T20/index.html) and AmiVital (http://www.amivital.es/index. php) projects. Tratamiento 2.0 is developing a middleware platform for the management and application of intelligent treatments, using EBM as knowledge base source. It tries to promote the delocalization in supervision and treatment delivery. Amivital is developing a technological framework that will allow the configuration of services and applications for patients, through an efficient use of available ambient intelligence technology.

Unfortunately, knowledge generation from EBM by means of intelligent agents and expert systems is still very immature, and thus, the use of expert and intelligent systems in healthcare, taking EBM as the source of knowledge is a non-solved issue yet.

\section{Synergy Between Smart Sensors and Integrative Research for E-Health}

To the best of our knowledge, the first synergistic use of smart sensors and integrative research for e-health was presented in [61]. Fig. (2) presents the information flow followed in the knowledge-based digital healthcare system presented in that work, which has been adapted to generalize the concept of patient image to healthy human image. As shown, knowledge discovering and processing follows three main layers, smart sensors, human physiological image (HPI) simulator, and clinical decision support system. It also shows the Remote Access Unit (RAU), which connects the assisted user to the services provider centre.

The smart sensors layer captures and processes biosignals from the associated user in a customized manner, according to the better performance of this approach [62]. These data feed mathematical models integrated into the HPI computational components. The technological architecture of these components, denoted previously as PPI, was described 


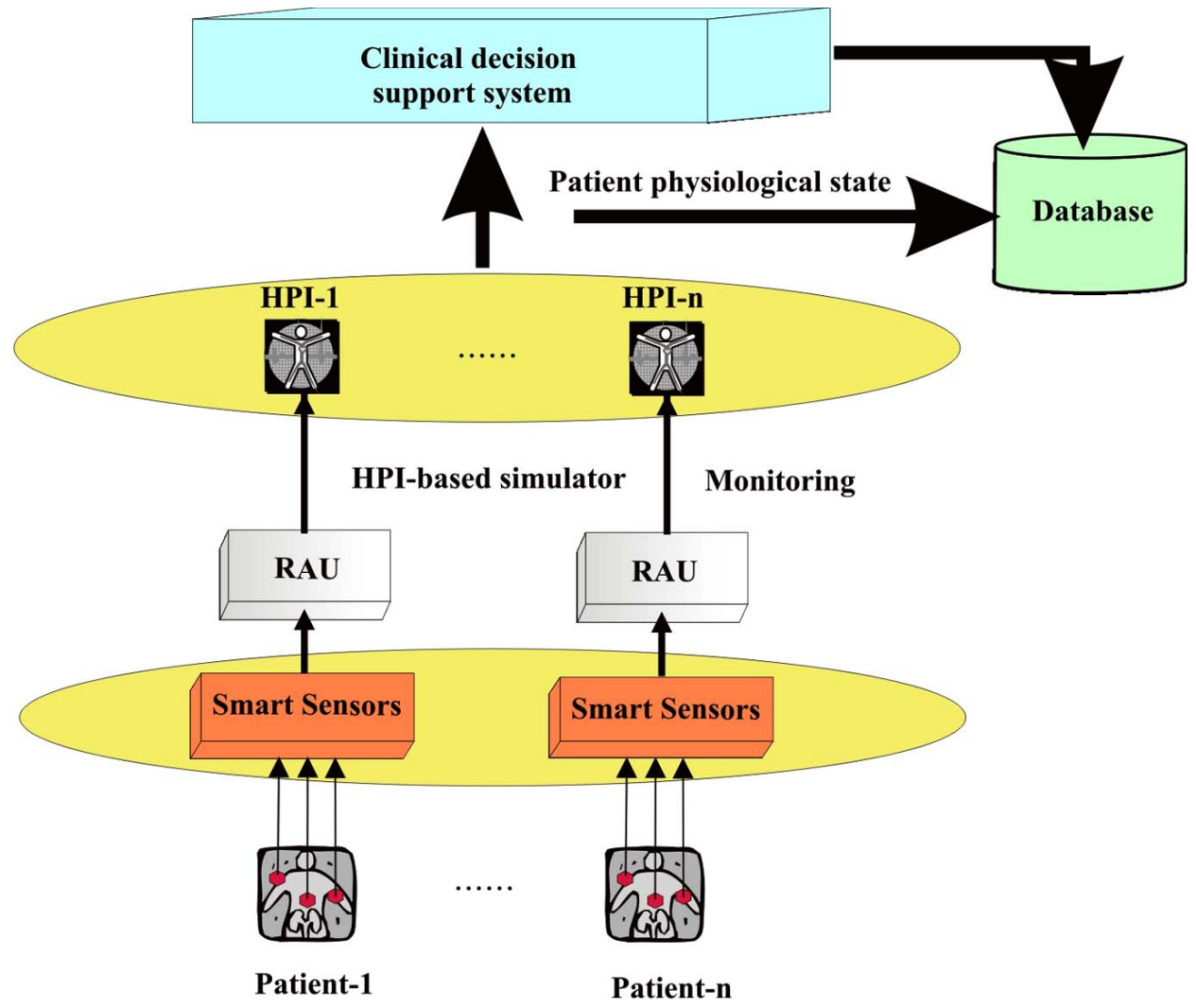

Fig. (2). Information flow followed in a knowledge-based digital healthcare system, adapted from [61].

in [61] and subsequently in [64]. The HPI simulator is an extension of the PPI (patient physiological image) presented in the cited work with the aim of extending the services from patients (pathologies) to healthy humans (prevention stage). HPIs integrate systemic multilevel mathematical models of those physiological aspects of the associated human that are relevant for the following up. Fig. (3) shows a simplistic block diagram focused on the mathematical model included into HPI and the signal loops that support the function of adapting the HPI to the changes of patient dynamics.

Accordingly, the HPI embedded into the second processing layer of Fig. (3) acts as a mathematical observer of the patient dynamics. Other instances of this mathematical model can be executed by means of HPI clones, starting from the current state to predict in a short-term the response of the patient to several actuations (drug intakes or changes in operative conditions of artificial organs like a hemodialyzer). This concept was evaluated in [61, 64] in the renal area, changing flow rates of an associated dialyzer (artificial kidney).

The same figure shows how internal biomedical variables computed by the model, are also identified and stored into an extension of the Electronic Health Record (EHR).

Fig. (4) presents an iconic diagram of a mathematical model that can be integrated into an HPI. This is an extension of the one that was published in [62], by considering now the pancreas organ separated from the muscular and other tissues block.

Figure tries to show the hierarchical and multilevel methodology that we are researching. Diagram (a) into Fig. (4) shows a pharmacokinetic mathematical model that is extended using hierarchical, object-oriented, and non-causal mathematical methodologies to integrate more detailed knowledge of several tissues. Diagrams (d-e) of Fig. (4) present also an iconic draw of an "average" nephron, together with the causal diagram concerning the control loops related to $\mathrm{L}_{\mathrm{p}}{ }^{*}$ (hydraulic permeability through aquaporin-2 channels) in the kidney collecting duct cells, at genetic (solid arrows), protein (dotted arrows) and subcellular level (dashed-dot arrows), where VP is vasopressin, $\mathrm{PKA}$ is protein kinase, $\mathrm{AQP} 2$ is aquaporin protein (and TAQP2 total AQP2). The consideration of this type of water specific channel in kidney ducts is very interesting in DM patients, because they could throw light on the evolution of renal disease in these population [65].

As indicated, Fig. (4) illustrates some details of the methodology followed in HPI models. As pharmacokinetic models can be considered simplified physiological models, we start from a whole body diagram (a) based on a pharmacokinetic approximation. This starting model can be extended considering anatomical aspects together with a multilevel extension of some subsystems, like organs and other groups of functional organic elements.

This manner, Fig. (4) extends the description of the pharmacokinetic renal system to describe the dynamics of renal cell groups into their basic functional units. This methodology requires an adequate technology which allows the re-use of submodels (simulation components) at different scales and levels. It is necessary the use of a non-causal modelling language to avoid incompatibilities among models developed through different methodologies. 


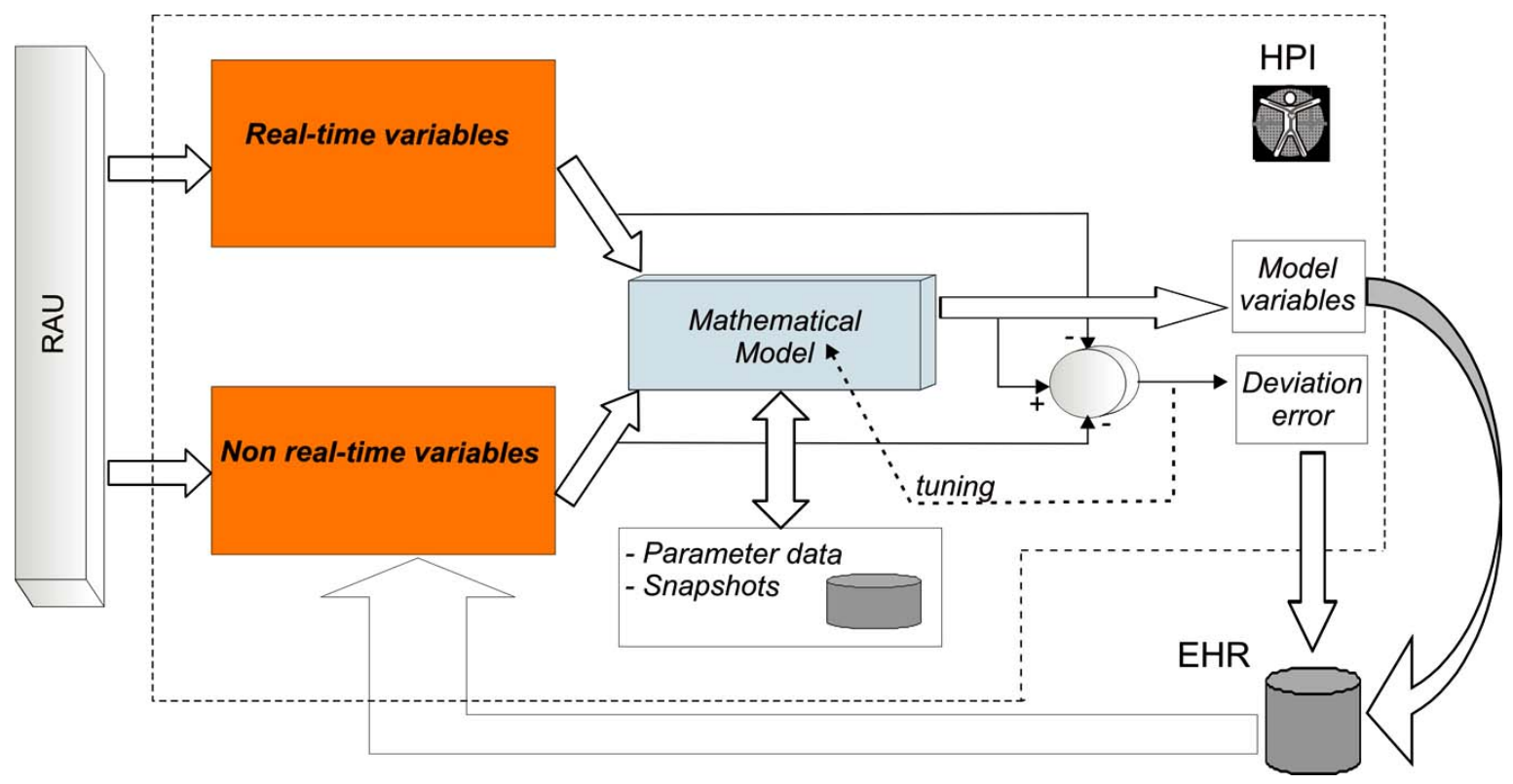

Fig. (3). Adaptive mathematical model included into the HPI component of the presented digital healthcare, adapted from [64].

Fig. (2) presents also a last and highest level processing layer that uses knowledge from HPI's layer to support the clinical decision. This processing layer is associated with driven-data models defined by expert systems, which in turn can integrate Evidence-based Medicine (EBM) and ambient intelligence sources (like Tratamiento 2.0 or Amivital projects, mentioned in Section 6.2).

Although EBM sources present methodological problems with respect to the manner in which they must be traduced to the clinical praxis and with respect to the integration into artificial intelligence subsystems [66-68], the inclusion of an intermediate and predictive layer based on HPIs could help to solve some of these problems. It takes into account that HPIs provide prediction based on causal mechanisms, whereas EBM sources complement this knowledge with scientific evidence non-based on true causal relationships but supported on statistical data.

The EHR database, as indicated in Fig. (3), requires an extension concerning to the type of information kept regarding the patient. This is a delicate issue, since EHR is the pivot over modern digital healthcare systems integrate patient-based knowledge, treatment following up, biomedical signal monitoring, and organizational and professional workflow. There is still no consensus regarding the manner to measure the effectivity of EHR on hospitals [69], and there is still difficulties concerning the fulfillment of clinical standards. Moreover, the information that must be added to EHR according to the methodology proposed in this work is very sensitive.

However, despite these difficulties, current tendencies on knowledge-based e-health systems point to the convergence between this area and integrative research based on Physiome and other Systems Biology initiatives. This convergence will promote the discovering of knowledge regarding the pathophysiology of DM, and it suggests an approach to achieve a true personalized medicine, with treatments oriented to the restoration or compensation of damaged physiological systems.

\section{DISCUSSION AND CONCLUSION}

The presented methodology shares objectives with the Physiome initiative, and helps to solve some of its lacks, thanks to the fusion of object-oriented hierarchy and mathematical non-causal modelling language paradigms into a telehealthcare approach.

Reusable multilevel physiological models are developed in the frame of this methodology, which avoids the mathematical decoupling of a system into its constituent mathematical submodels, each one described in the proper functional hierarchical level. Therefore, our methodology allows a true multilevel description of strongly coupled subsystems.

This is an issue still non-solved in Physiome, where system models pertaining to different organizational living levels are connected via tools like the System Biology Workbench (SBW), impeding a strong coupling among them.

We use standards for the exchange of information required to achieve the cooperative use of different models and adopt a standard nomenclature based on formal ontologies for model components, in agreement with the Physiome initiative.

It is worth to note that it is necessary an effort to connect modelling languages selected in the architecture depicted in this article with those used in Physiome. For this objective, an XML-based language (ELML) for coding mathematical models based on our multilevel modelling methodology, as well as a conversion tool between the JSim's Mathematical Modelling Language (MML) used in Physiome and ELML, are being developed. Mathematical models in ELML need to be converted to EcosimPro Language (EL) previously to be executed. This tool will strongly boost the diffusion of our methodology among research groups working in Physiome VPH initiatives.

Smart sensors play a key role in the computational architecture presented, as they constitute the first processing 


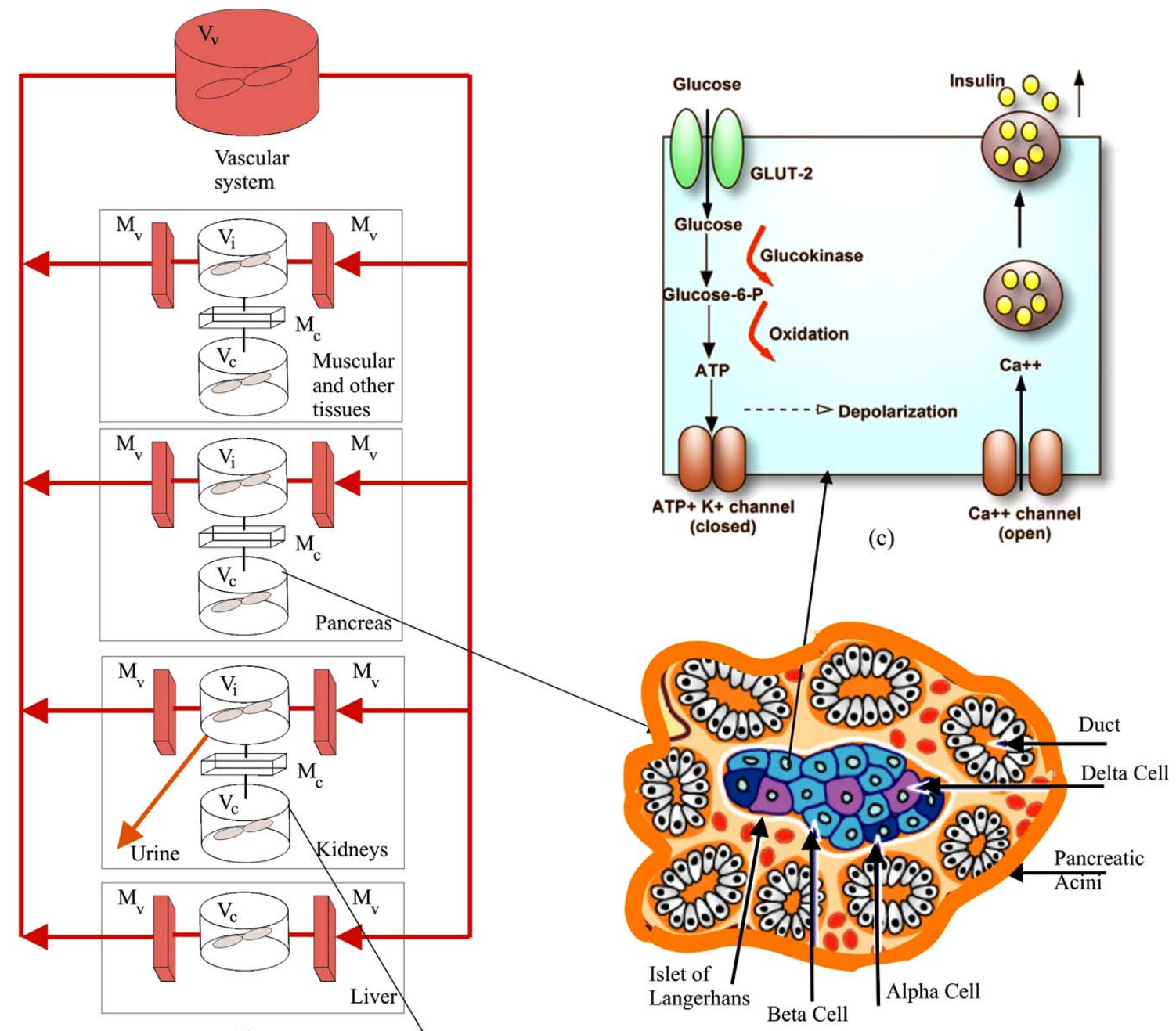

(a)

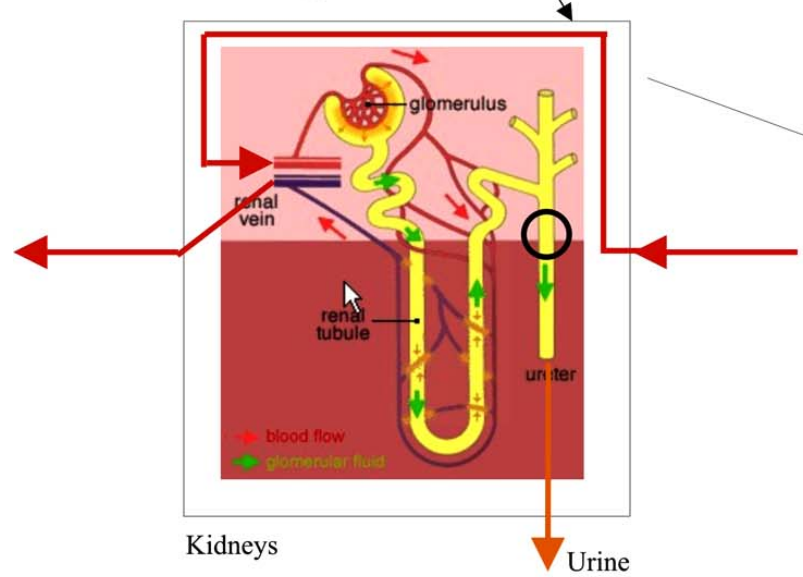

(b)

(d)

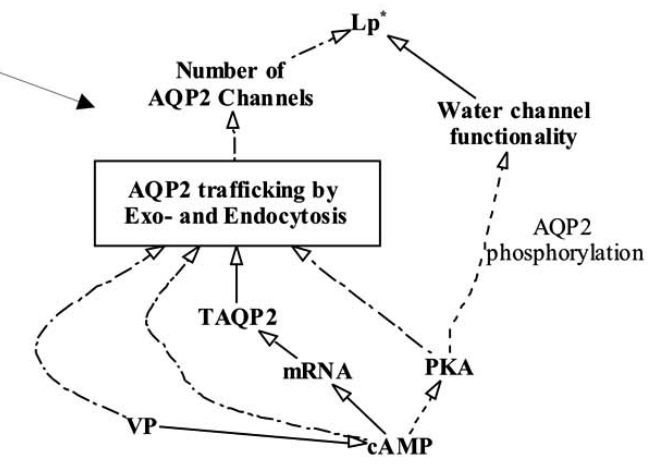

(e)

Fig. (4). Iconic diagrams of a mathematical model integrated into an HPI. Extended from [62].

layer of knowledge generation. Smart sensors acquire biomedical inputs, process them in a customized and distributed manner, and transmit information to the HPI's layer. The adaptive feature of smart sensors is also critical, with the aim of providing them the ability to evolve together with the linked user and their environment, in a reactive and transparent manner. Accordingly, these sensors provide ambient intelligence to the system.
There is a strong need to seek methodologies and technologies which can bring added values to e-health system and facilitate the discovering and management of customized biomedical knowledge regarding the assisted users. This fact supports the change of paradigm in healthcare, from the current centralized model focused to the illness to a truly personalized, preventive, predictive, and participatory (4P) healthcare model focused to the health. 
An advantage of such change of paradigm is the transfer of many of Physiome goals to telehealthcare environments, which will strongly speed up the flux of knowledge between clinical and researchers.

Notwithstanding Physiome and VPH initiatives, as tools to characterize the biomedical knowledge of humans, are currently in a preliminary stage. It is necessary an international effort to advance in this task. We claim that the hybridizing VPH/Physiome - based initiatives with knowledge discovering and managing technologies for ehealth, as we do with the HPI concept, will push the maturation of both areas and will improve the life expectancy and quality of life of the citizens.

\section{ACKNOWLEDGEMENTS}

This work has been partly supported by the Dirección General de Investigación y Gestión del Plan Nacional de I+D+i, Ministerio de Ciencia e Innovación, under Grant TIN2009-13981 (TSI).

\section{REFERENCES}

[1] A. Makroglou, J. Li, and Y. Kuang, "Mathematical models and software tools for the glucose-insulin regulatory system and diabetes: an overview," Appl. Numer. Math., vol. 56, pp. 559-573, 2006.

[2] A. C. Guyton, and J. E. Hall, Textbook of Medical Physiology, Eleventh Edition ed.: Elsevier Saunders, 2006.

[3] B. K. Tripathi, and A. K. Srivastava, "Diabetes mellitus: complications and therapeutics," Med. Sci. Monit., vol. 12, pp. RA130-47, July 1, 2006.

[4] WHO Consultation, "Definition, Diagnosis and Classification of Diabetes Mellitus and its Complications," WHO WHO/NCD/NCS/99.2, 1999.

[5] M. Haag, and N. G. Dippenaar, "Dietary fats, fatty acids and insulin resistance: short review of a multifaceted connection," Med. Sci. Monit., vol. 11, pp. RA359-367, 2005.

[6] J. Shepherd, "Dyslipidaemia in diabetic patients: time for a rethink," Diabetes Obes. Metab., vol. 9, pp. 609-616, 2007.

[7] Diabetes Action Now Initiative, "Diabetes Action Now: an iniative of the World Health Organization and the International Diabetes Federation," WHO and IDF, Switzerland 2004.

[8] S. Wild, G. Roglic, A. Green, R. Sicree, and H. King, "Global Prevalence of Diabetes: Estimates for the year 2000 and projections for 2030," Diabetes Care, vol. 27, pp. 1047-1053, May 1, 2004.

[9] C. J. Green, P. Fortin, M. Maclure, A. Macgregor, and S. Robinson, "Information system support as a critical success factor for chronic disease management: Necessary but not sufficient," Int. J. Med. Inf., vol. 75, pp. 818-828, 2006.

[10] E. R. Carson, D. G. Cramp, A. Morgan, and A. V. Roudsari, "Clinical decision support, systems methodology, and telemedicine: their role in the management of chronic disease," IEEE Trans. Inf. Technol. Biomed., vol. 2, pp. 80-8, 1998.

[11] A. J. Farmer, O. J. Gibson, C. Dudley, K. Bryden, P. M. Hayton, L. Tarassenko, and A. Neil, "A randomized controlled trial of the effect of real-time telemedicine support on glycemic control in young adults with type 1 diabetes (ISRCTN 46889446)," Diabetes Care, vol. 28, pp. 2697-2702, November 1, 20052005.

[12] J. C. Wei, D. J. Valentino, D. S. Bell, and R. S. Baker, "A webbased telemedicine system for diabetic retinopathy screening using digital fundus photography," Telemed. J. Health, vol. 12, pp. 50-57, 2006.

[13] J. M. Medina, F. S. d. Medina, and A. Vargas, Bioquímica. Madrid: Editorial Síntesis, 1996.

[14] S. Kauffman, Investigaciones. Barcelona: Tusquet Editores, 2003.

[15] A. Hintze, and C. Adami, "Evolution of complex modular biological networks," PLoS Comput. Biol., vol. 4, p. e23, February 01, 2008.

[16] A. Aderem, "Systems biology: Its practice and challenges," Cell, vol. 121, pp. 511-513, 2005.
[17] A. J. O'Sullivan, and J. J. Kelly, "Insulin resistance and protein catabolism in non-diabetic hemodialysis patients," Kidney Int., vol. 71, pp. 98-100, 2006.

[18] R. Bertram, A. Sherman, and L. S. Satin, "Metabolic and electrical oscillations: partners in controlling pulsatile insulin secretion," Am. J. Physiol. Endocrinol. Metab., vol. 293, pp. E890-900, October 1, 2007.

[19] G. Grodsky, "A threshold distribution hypothesis for packet storage of insulin and its mathematical modeling," J. Clin. Investig., vol. 51, pp. 2047-2059, 1972.

[20] V. Licko, "Threshold secretory mechanism: a model of derivative element in biological control," Bull. Math. Biol., vol. 35, pp. 51-58, 1973.

[21] E. Cerasi, G. Fick, and M. Rudemo, "A mathematical model for the glucose induced insulin release in man," Eur. J. Clin. Investig., vol. 4, pp. 267-278, 1974.

[22] G. Toffolo, R. Bergman, D. Finegood, C. Bowden, and C. Cobelli, "Quantitative estimation of beta cell sensitivity to glucose in the intact organism: a minimal model of insulin kinetics in the dog," Diabetes, vol. 29, pp. 979-990, 1980.

[23] B. Topp, K. Promislow, G. deVries, R. Miura, and D. Finegood, "A model of beta-cell mass, insulin, and glucose kinetics: pathways to diabetes," J. Theor. Biol., vol. 206, pp. 605-19, 2000.

[24] R. Hernandez, D. Lyles, D. Rubin, T. Voden, and S. Wirkus, "A model of beta-cell mass, insulin, glucose, and receptor dynamics with applications to diabetes," Cornell University Department of Biological Statistics and Computational Biology Technical Report, BU-1579-M, 2001

[25] P. Maechler, and C. B. Wollheim, "Mitochondrial glutamate acts as a messenger in glucose-induced insulin exocytosis," Nature, vol. 402, pp. 685-689, 1999.

[26] R. Bertram, and A. Sherman, "A calcium-based phantom bursting model for pancreatic islets," Bull. Math. Biol., vol. 66, pp. 13131344, 2004.

[27] R. Bergman, Y. Ider, C. Bowden, and C. Cobelli, "Quantitative estimation of insulin sensitivity," Am. J. Physiol., vol. 236, pp. E667-77, 1979.

[28] H. Himsworth, and R. Ker, "Insulin-sensitive and insulin insensitive types of diabetes mellitus," Clin. Sci., vol. 4, pp. 119122, 1939.

[29] V. Bolie, "Coefficients of normal blood glucose regulation," $J$. Appl. Physiol., vol. 16, pp. 783-788, 1961.

[30] M. Derouich, and A. Boutayeb, "The effect of physical exercise on the dynamics of glucose and insulin," J. Biomech., vol. 35, pp. 911917, 2002.

[31] A. De Gaetano, and O. Arino, "Mathematical Modelling of the Intravenous Glucose Tolerance Test," J. Math. Biol., vol. 40, pp. 136-168, 2000.

[32] A. De Gaetano, and O. Arino, "A statistical approach to the determination of stability for dynamical systems modelling physiological processes," Math. Comput. Model., vol. 31, pp. 41$51,2000$.

[33] A. Mukhopadhyay, A. De Gaetano, and O. Arino, "Modelling the intravenous glucose tolerance test: A global study for singledistributed- delay model," Discr. Cont. Dyn. Syst. Ser. B, vol. 4, pp. 407-417, 2004.

[34] Z. Lam, K. Hwang, J. Lee, J. Chase, and G. Wake, "Active insulin infusion using optimal and derivative-weighted control," Med. Eng. Phys., vol. 24, pp. 663-672, 2002.

[35] G. Nucci, and C. Cobelli, "Models of subcutaneous insulin kinetics. A critical review," Comp. Meth. Progr. Biomed., vol. 62, pp. 249257, 2000.

[36] M. Prado, and L. Roa, "Simulation Languages," In Wiley Encyclopedia of Biomedical Engineering, A. M, Ed.: John Wiley and Sons, Inc, 2006, pp. 41-52.

[37] A. Marée, R. Kublik, D. Finegood, and L. Edelstein-Keshet, "Modelling the onset of Type 1 diabetes: can impaired macrophage phagocytosis make the difference between health and disease?," Philos. Transact. A Math. Phys. Eng. Sci., vol. 364, pp. 1267-1282, 2006.

[38] J. Hetherington, I. Bogle, P. Saffrey, O. Margoninski, L. Li, M. Varela-Rey, S. Yamaji, S. Baigent, J. Ashmore, K. Page, R. Seymour, A. Finkelstein, and A. Warner, "Addressing the challenges of multiscale model management in systems biology," Comput. Chem. Eng., vol. 31, pp. 962-979, 2007. 
[39] M. Hucka, A. Finney, H. Sauro, H. Bolouri, J. Doyle, and H. Kitano, "The ERATO Systems Biology Workbench: enabling interaction and exchange between software tools for computational biology," Pac. Symp. Biocomput., pp. 450-61, 2002.

[40] D. Watne, P. Turney, and D. Watne, Auditing EDP Systems, 2002.

[41] A. Elstein, "On the origins and development of evidence-based medicine and medical decision making," Inflamm. Res., vol. 53, pp. 184-189, 2004.

[42] A. Jacobson, M. de Groot, and J. Samson, "Quality of life research in patients with Diabetes Mellitus," in Quality if Life in Behavior Medicine Research, J. Dimsdale and A. Baum, Eds.: Lawrence Erlbaum Associates, 1995.

[43] DCCT Research Group, "Reliability and validity of a diabetes quality of life measure for the diabetes control and complication trial (DCCT)." Diabetes Care, vol. 11, pp. 725-732, 1988.

[44] C. Lloyd, K. Matthews, R. Wing, and T. Orchard, "Psychosocial factors and complications of IDDM," Diabetes Care, vol. 15, pp. 166-172, 1992.

[45] J. Selam, P. Micossi, and F. Dunn, "Clinical trial of programmable implantable insulin pump for Type I diabetes," Diabetes Care, vol. 15, pp. 877-885, 1992.

[46] J. Ware, and C. Sherbourne, "The MOS 36-Item Short Form From Health Survey (SF-36). Conceptual framework and item selection," Med. Care, vol. 30, pp. 473-483, 1992.

[47] K. Wells, J. Golding, and M. Burnam, "Psychiatric disorder in a sample of the general population with and without chronic medical conditions," Am. J. Psychiatry, vol. 145, pp. 976-981, 1988.

[48] S. M. Marshall, and A. Flyvbjerg, "Prevention and early detection of vascular complications of diabetes," BMJ (Clinical Research Ed.), vol. 333, pp. 475-480, September 2, 2006.

[49] A. T. Hattersley, and E. R. Pearson, "Minireview: pharmacogenetics and beyond: the interaction of therapeutic response, \{beta\}-cell physiology, and genetics in diabetes," Endocrinology, vol. 147, pp. 2657-2663, June 1, 2006.

[50] E. Codner, S. Flanagan, S. Ellard, H. Garcia, and A. T. Hattersley, "High-dose glibenclamide can replace insulin therapy despite transitory diarrhea in early-onset diabetes caused by a novel R201L Kir6.2 mutation," Diabetes Care, vol. 28, pp. 758-759, March 1, 2005.

[51] T. Klupa, E. Edghill, J. Nazim, J. Sieradzki, S. Ellard, A. Hattersley, and M. Malecki, "The identification of a R201H mutation in KCNJ11, which encodes Kir6.2, and successful transfer to sustained-release sulphonylurea therapy in a subject with neonatal diabetes: evidence for heterogeneity of beta cell function among carriers of the R201H mutation," Diabetologia, vol. 48, pp. 1029-31, May 1, 2005.

[52] A. Zung, B. Glaser, R. Nimri, and Z. Zadik, "Glibenclamide Treatment in Permanent Neonatal Diabetes Mellitus due to an Activating Mutation in Kir6.2," J. Clin. Endocrinol. Metab., vol. 89, pp. 5504-5507, November 1, 2004.

[53] M. T. Malecki, J. Skupien, T. Klupa, K. Wanic, W. Mlynarski, A. Gach, I. Solecka, and J. Sieradzki, "Transfer to sulphonylurea therapy in adult subjects with permanent neonatal diabetes due to KCNJ11-activating mutations: evidence for improvement in insulin sensitivity," Diabetes Care, vol. 30, pp. 147-149, January 1, 2007.

[54] G. Knopf, and A. Bassi, Smart Biosensor Technology, 2006.
[55] M. Prado-Velasco, M. G. d. Río-Cidoncha, and R. Ortíz-Marín, "The Inescapable Smart Impact detection System (ISIS): an ubiquitous and personalized fall detector based on a distributed "divide and conquer strategy"," in Personalized Healthcare through Technology, 30th Annual International Conference of the IEEE Engineering in Medicine and Biology Society Vancouver, British Columbia, Canada, pp. 3332-3335, 2008.

[56] J.-L. Coatrieux, and J. Bassingthwaighte, "Special issue on the physiome and beyond," Proc. IEEE, vol. 94, pp. 671-677, 2006.

[57] P. Hunter, P. Robbins, and D. Noble, "The IUPS human physiome project," Eur. J. Physiol., vol. 445, pp. 1-9, 2002.

[58] D. Ngon, J. M. Patrick, and C. F. Dewey, "The human physiome as an information environment," Ann. Biomed. Eng., vol. 28, p. 1032, 2000.

[59] J. D. Bronzino, "The biomedical engineering handbook," in The Electrical engineering handbook series, 2nd ed. vol. 2 Florida: CRC Press, p. 1512, 2000.

[60] P. J. Hunter, "Modeling human physiology: The IUPS/EMBS physiome project," Proc. IEEE, vol. 94, pp. 678-691, 2006.

[61] M. Prado, L. Roa, J. Reina-Tosina, A. Palma, and J. A. Milán, "Virtual center for renal support: technological approach to Patient Physiological Image," IEEE Trans. Biomed. Eng., vol. 49, pp. 1420-1430, Dec 2002.

[62] M. Prado, L. M. Roa, and J. Reina-Tosina, "Viability study of a personalized and adaptive knowledge-generation telehealthcare system for nephrology (NEFROTEL)," Int. J. Med. Inf., vol. 75, pp. 646-657, 2006.

[63] L. Hood, "Systems Biology and Systems Medicine: From Reactive to Predictive, Personalized, Preventive and Participatory Medicine," in Personalized Healthcare through Technology, 30th Annual International Conference of the IEEE Engineering in Medicine and Biology Society Vancouver, British Columbia, Canada, 2008.

[64] M. Prado, L. Roa, J. Reina-Tosina, A. Palma, and J. A. Milán, "Renal telehealthcare system based on a patient physiological image: a novel hybrid approach in telemedicine," Telemed. J. Health, vol. 9, pp. 149-165, 2003.

[65] P. Bardoux, M. Ahloulay, S. Le Maout, L. Bankir, and M. TrinhTrang-Tan, "Aquaporin-2 and urea transporter-A1 are up-regulated in rats with type I diabetes mellitus," Diabetologia, vol. 44, pp. 637-45, May 1, 2001.

[66] J. P. Briggs, "Evidence-Based medicine in the dialysis unit: a few lessons from the USRDS and the NCDS and HEMO trials," Semin. Dial., vol. 17, pp. 136-141, 2004.

[67] M. Jenicek, "Evidence-based medicine: Fifteen years later. Golem the good, the bad, and the ugly in need of a review?," Med. Sci. Monit., vol. 12, pp. RA241-251, 2006.

[68] S. Rosenbaum, C. Glenton, and J. Cracknell, "User experiences of evidence-based online resources for health professionals: User testing of The Cochrane Library," BMC Med. Inf. Decis. Mak., vol. 8, p. 34, 2008.

[69] G. O. Otieno, T. Hinako, A. Motohiro, K. Daisuke, and N. Keiko, "Measuring effectiveness of electronic medical records systems: Towards building a composite index for benchmarking hospitals," Int. J. Med. Inf., vol. 77, pp. 657-669, 2008.

(C) Peruchena and Prado-Velasco; Licensee Bentham Open.

This is an open access article licensed under the terms of the Creative Commons Attribution Non-Commercial License (http://creativecommons.org/licenses/by-nc/3.0/) which permits unrestricted, non-commercial use, distribution and reproduction in any medium, provided the work is properly cited. 